\title{
Avaliação da qualidade de sono em estudantes de medicina de uma instituição de ensino superior de Teresina
}

\author{
Evaluation of sleep quality in medicine students in a university in Teresina \\ Evaluación de la calidad del sueño en estudiantes de medicina de una institución de \\ educación superior en Teresina
}

André Lucas de Oliveira Andrade Silveira ${ }^{1}$, Luciano Pires Vilanova ${ }^{1 *}$, Karícia Lima de Freitas Bonfim ${ }^{1}$, Clara Dantas da Fonseca ${ }^{1}$, Bruno Gusmão Menezes de Aguiar ${ }^{1}$, João Luiz Vieira Ribeiro ${ }^{1}$.

\section{RESUMO}

Objetivo: Descrever a qualidade de sono de estudantes do curso de Medicina numa instituição de ensino superior de Teresina-PI e correlacioná-la com dados epidemiológicos. Métodos: O estudo é do tipo transversal analítico, realizado através de questionário autoaplicável online contendo o validado Índice de Qualidade do Sono de Pittsburgh (PSQI), além de dados sociodemográficos adicionais. Resultados: A amostra foi composta por 151 alunos. De acordo com o PSQI, 79,48\% $(n=120)$ dos participantes apresentavam qualidade ruim no padrão do sono. Houve significância estatística apenas entre sono de qualidade ruim e uso de medicações $(p<0,05)$. Conclusão: Percebe-se que, apesar da extensa literatura abrangendo a qualidade de sono nessa população, pouco se faz para combater esse sério problema. A má qualidade de sono continua sendo negligenciada, principalmente pelos próprios estudantes, que não se reconhecem como população de risco e não buscam, na maioria das vezes, a correção de hábitos ou atitudes prejudiciais que comprometem a qualidade do sono.

Palavras-chave: Estudantes de medicina, Sono, Transtornos do sono-vigília.

\begin{abstract}
Objective: To describe the quality of sleep of medical students at na university in Teresina-PI and correlate it with epidemiological data. Methods: The study is a cross-sectional analytical type, carried out through an online selfadministered questionnaire containing the validated Pittsburgh Sleep Quality Index (PSQI), in addition to additional socio-demographic data. Results: The sample consisted of 151 students. According to the PSQI, 79.48\% $(n=120)$ of the participants had poor quality sleep patterns. There was statistical significance only between poor quality sleep and medication use $(p<0.05)$. Conclusion: It is clear that, despite the extensive literature covering the quality of sleep in this population, little is done to combat this serious problem. The poor quality of sleep continues to be neglected, mainly by the students themselves, who do not recognize themselves as a population of risk and do not seek, in most cases, the correction of harmful habits or attitudes that compromise their quality of sleep.
\end{abstract}

Keywords: Medical students, Sleep, Sleep wake disorders.

\section{RESUMEN}

Objetivo: Describir la calidad del sueño de los estudiantes de medicina de una institución de educación superior en Teresina-PI y correlacionarla con datos epidemiológicos. Métodos: El estudio es de tipo analítico transversal, realizado a través de un cuestionario autoadministrado en línea que contiene el Índice de Calidad del Sueño de Pittsburgh (PSQI) validado, además de datos sociodemográficos adicionales. Resultados: La muestra estuvo conformada por 151 estudiantes. Según el PSQI, el 79,48\% $(n=120)$ de los participantes tenían patrones de sueño de mala calidad. Sólo hubo significación estadística entre la mala calidad del sueño y el uso de medicamentos ( $p$ $<0,05)$. Conclusión: es evidente que, a pesar de la extensa literatura que cubre la calidad del sueño en esta población, poco se hace para combatir este grave problema. La mala calidad del sueño sigue siendo desatendida, principalmente por los propios estudiantes, que no se reconocen como población de riesgo y no buscan, en la mayoría de los casos, la corrección de hábitos o actitudes nocivas que comprometan la calidad del sueño.

Palabras clave: Estudiantes de medicina, Sueño, Trastornos del sueño y vigília.

\footnotetext{
${ }^{1}$ Centro Universitário UNINOVAFAPI, Teresina - PI. *E-mail: Ipiresvilanova@gmail.com
} 


\section{INTRODUÇÃO}

O ciclo sono-vigília é um dos principais fenômenos fisiológicos do nosso organismo, pois consegue influenciar componentes físicos, hormonais e psicológicos de uma pessoa. Sendo afetado naturalmente por fatores externos (como alternância do dia-noite (claro-escuro) e condições do local em que se dorme) e fatores internos (como estímulos no núcleo supraquiasmático do hipotálamo) funcionando em um ritmo circadiano (SEGUNDO LVG, et al.,2017). O equilíbrio entre estes fatores é essencial para o funcionamento adequado do fenômeno de ciclo sono-vigília, e, por conseguinte, para a manutenção da saúde do corpo humano (SEGUNDO LVG, et al.,2017; RIBEIRO CR, et al.,2014).

Entretanto, na sociedade atual, outros fatores têm assumido papel cada vez maior no seu funcionamento, tais como estilo de vida do indivíduo, horários escolares, de trabalho ou de lazer, além de fatores psicológicos, que podem alterar o funcionamento correto desse ciclo e afetar a qualidade de sono e a saúde, pois pessoas que dormem mal tendem a ter mais morbidades, menor expectativa de vida e envelhecimento precoce (CORRÊA CC, et al., 2017; SEGUNDO LVG, et al.,2017).

As consequências dos distúrbios de sono se desdobrarão em pelo menos três níveis seguidos que comprometerão a qualidade de vida do indivíduo acometido. Num primeiro nível, encontram-se as variações biológicas, as quais trarão consequências mais imediatas ao organismo, como a fadiga, falhas de memória, dificuldade de atenção, hipersensibilidade, distúrbios de humor e Burnout. Á médio prazo, os aspectos funcionais serão prejudicados, tais como o aumento do absenteísmo e risco de acidentes, prejuízo nas relações de trabalho, familiares e sociais e diminuição de desempenho nos estudos. Por fim, serão observados a longo prazo ocorrências como demissões e problemas de saúde (FERREIRA CMG, et al.,2017; HIROTSU C, et al., 2014).

Esse achado é significativo do ponto de vista da saúde pública, porque o sono insuficiente entre futuros profissionais de saúde pode prejudicar o desempenho e as demandas de seu trabalho. Isso é particularmente prejudicial na profissão médica, onde o desempenho prejudicado dos profissionais de saúde privados de sono pode levar a conseqüências desastrosas no atendimento ao paciente (AI-KANDARI S, et al., 2017). No Brasil, estudo realizado em 132 cidades constatou que $76 \%$ dos participantes, com idade de 16 anos ou mais, apresentaram algum problema relacionado ao sono, os mais comuns sendo sono insuficiente e ronco (HIROTSU C, et al., 2014).

Logo, é natural pensar que exista uma maior prevalência de distúrbios do sono entre os estudantes de medicina, principalmente devido a fatores como a ocorrência de aulas em período integral, necessidade de grande demanda de tempo para atividades extracurriculares (estágios, pesquisas científicas, projetos de extensão, monitorias) e ainda dedicar-se aos estudos (OKANO K, et al., 2019).

Qualidade do sono, duração e consistência juntos representaram uma quantidade substancial (cerca de um quarto) da variação geral no desempenho acadêmico (OKANO K, et al., 2019). Além de outros agravantes, como hábito alimentar insatisfatório, ausência de atividade física regular e ansiedade/angústia. Visto que os objetivos de trabalho do futuro profissional médico terão como base o atendimento das necessidades de prevenção, promoção ou recuperação do estado de saúde do ser humano, faz-se necessário olhar mais atentamente para o seu bem-estar durante a formação desses profissionais (CHAZAN ACS, et al., 2015).

Diante do exposto, o objetivo deste estudo foi avaliar a percepção subjetiva da qualidade de sono em estudantes de Medicina, comparando as fases do curso, além das variáveis epidemiológicas sexo, idade e prática de atividades.

\section{MÉTODOS}

Trata-se de um estudo do tipo analítico transversal, no qual foram convidados 882 alunos do curso de graduação em medicina, do primeiro ao oitavo período, de uma instituição de Teresina-PI. A coleta de dados foi realizada durante três semanas. O estudo foi aprovado pelo comitê de ética em pesquisa da instituição, de acordo com a Resolução CNS 466/12 (CAAE:28748920.9.0000.5210). Todos os participantes aceitaram o termo de consentimento livre e esclarecido antes de responder o questionário da pesquisa. Os alunos que não aceitaram os termos da pesquisa foram excluídos. 
A escolha do questionário de pesquisa índice de qualidade de sono de Pittsburgh (PSQI) vê-se ao fato de que esse é um instrumento de análise difundido mundialmente e validado para a avaliação de distúrbios do sono. O questionário foi desenvolvido por Buysse DJ, et al. (1989), sendo composto por 19 perguntas autoadministradas, referentes à qualidade e distúrbios de sono no último mês, as quatro primeiras escritas e as outras 15 de múltipla escolha. $O$ questionário avalia sete componentes do sono: qualidade subjetiva, latência do sono, duração do sono, eficiência do sono, distúrbios do sono, uso de medicamento se disfunção diária. Para cada componente a pontuação pode variar de 0 a 3, e soma total dos 7 componentes pode variar num escore de 0 a 21 pontos. Escore acima de 5 pontos implicam em qualidade de sono ruim, indicando possíveis distúrbios do sono.

Os questionários foram respondidos de forma online através da plataforma Google Forms e seus dados foram computados em tabelas com o uso dos programas Google Sheets, Microsoft Excel e Statistical Package for Social Science (SPSS), com os resultados da amostra geral e outras separadas por ano de graduação, $1^{\circ}$ ao $4^{\circ}$ anos de graduação, sexo, idade e frequência de atividade física. A opção de incluir apenas alunos o $1^{\circ}$ ao $4^{\circ}$ ano se deu ao fato destes estarem cursando as disciplinas de formação básica geral e as de formação específica, assim, constituindo um grupo mais homogêneo com relação ao cotidiano acadêmico. Foi realizada a análise pelas proporções das diferentes respostas em função de cada componente.

$\mathrm{Na}$ análise, foi utilizado o Teste Qui-Quadrado e Teste G para a comparação na amostra geral e nos grupos de estudantes com os sete componentes do PSQI. Índice de Confiança de 95\%. O nível de significância utilizado foi de $5 \%$. Valores de $p>0,05$ foram considerados não significativos.

\section{RESULTADOS}

Do total de 882 alunos convidados para participar do estudo, 151 (17,1\%) responderam o PSQI, agrupados, em ordem crescente do $1^{\circ}$ ao $4^{\circ}$ anos, em 66, 29, 20 e 36 alunos, respectivamente. Em relação ao gênero, foi verificada uma predominância na proporção de mulheres.

De acordo com o PSQI, 79,48\% $(n=120)$ dos participantes apresentavam qualidade ruim no padrão do sono. Nota-se no gráfico 1 que a maioria dos alunos apresenta uma qualidade ruim de sono em todos os anos da graduação avaliados. Não foi encontrada relação entre a qualidade do sono e o gênero dos estudantes, assim como não houve relação com o ano da graduação (Tabela 1).

Gráfico 1 - Qualidade do sono de acordo com o ano da graduação.

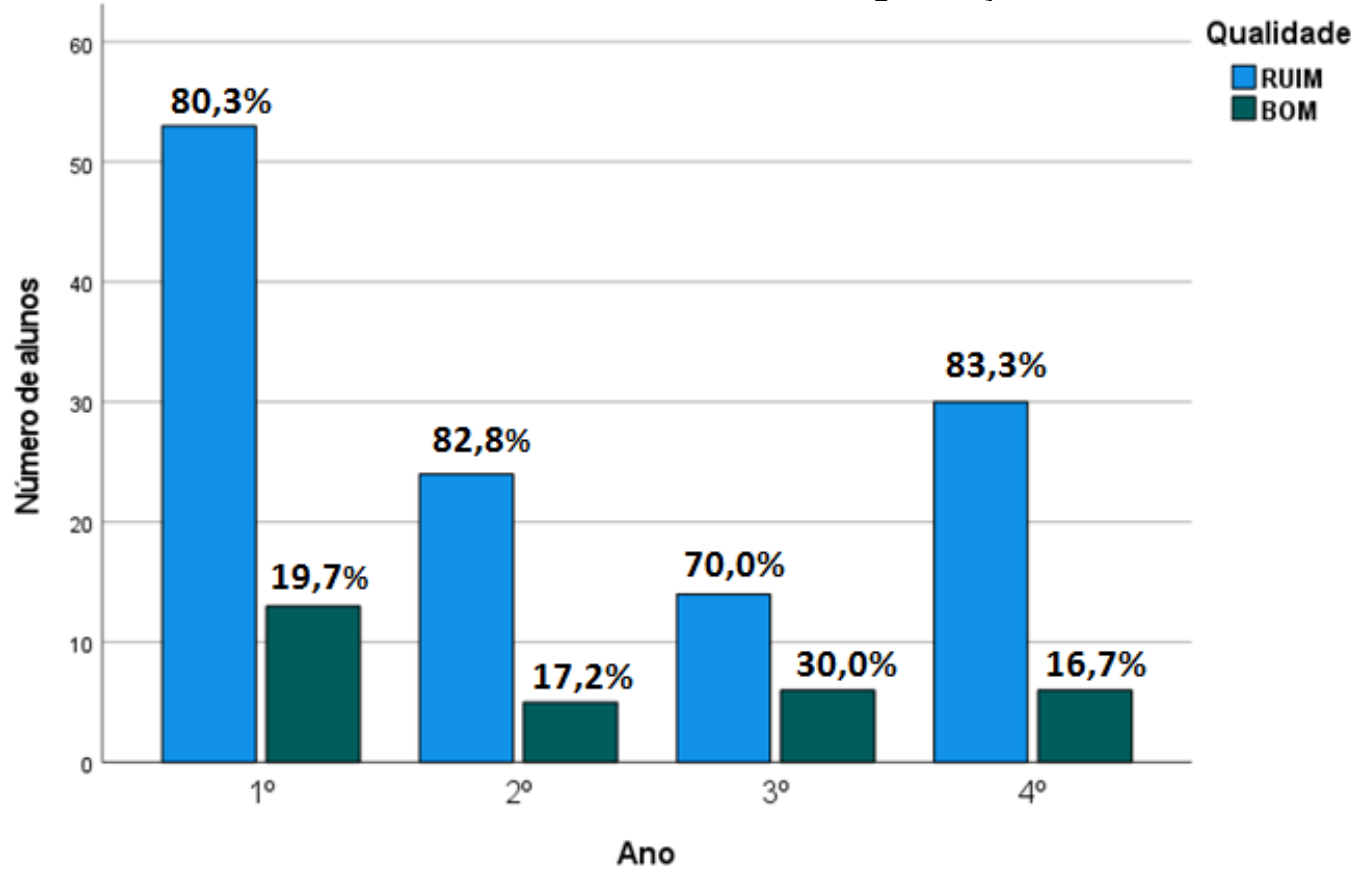

Fonte: Silveira ALOA, et al., 2021. 
Com relação à idade, os participantes foram distribuídos em 2 grupos. Entre os alunos com idade menor ou igual 25 anos verificou-se que $63,6 \%(n=96)$ apresentaram qualidade de sono ruim e nos alunos acima dessa faixa etária foi de $16,5 \%(n=25)$. Após análise estatística, não foi constatada correlação significativa entre a idade e qualidade de sono (Tabela 1).

Quanto à frequência de atividade física no mês, 24,5\% $(n=37)$ dos participantes que afirmaram não praticar nenhuma atividade física foram classificados com qualidade ruim de sono, e entre os que afirmaram praticar atividade física 3 ou mais vezes por semana o valor encontrado foi de $28,5 \%(n=43)$. Logo, não foram observadas diferenças estatisticamente significantes entre a prática de atividade física e qualidade do sono (Tabela 1).

Os resultados do PSQI foram analisados na amostra geral com seus sete componentes. Quanto à avaliação da própria qualidade sono, $6 \%(n=9)$ dos estudantes classificaram como muito boa, $49 \%(n=74)$ como boa, $40,4 \%(n=61)$ como ruim e $4,6 \%(n=7)$ como muito ruim. $33,1 \%$ afirmaram demorar mais de 30 min para conseguir dormir pelo menos uma vez por semana. 31,1\% afirmaram que dormiam menos de 6 horas por noite. Esses dados se referem, respectivamente, à qualidade subjetiva do sono, à latência do sono e à duração do sono.

A eficiência habitual do sono foi considerada adequada em $72,2 \%(n=109)$ da amostra geral. Apenas $2 \%$ $(n=3)$ não responderam esse item corretamente, logo não foram incluídos nesta análise. No disposto à frequência de distúrbios do sono, esta foi considerada baixa em $68,2 \%(n=103)$ da amostra geral. $\mathrm{Na}$ análise estatística, ambos os componentes não apresentaram diferenças significativas.

Foi observado ainda que $23,9 \%(n=36)$ dos entrevistados afirmaram tomar medicamentos para dormir, e dentre estes, todos foram classificados com sono ruim. É possível verificar correlação entre uso de medicação para dormir e qualidade de sono ruim $(p=0,006)$.

Em relação à disfunção durante o dia, 23,3\% $(n=35)$ relataram apresentar dificuldades em se manter acordado durante o dia pelo menos uma vez por semana e $54,0 \%(n=83)$ relataram ao menos uma dificuldade razoável em manter o ânimo para as atividades habituais diária.

Tabela 1- Relação das variáveis estudadas e significância estatística.

\begin{tabular}{|c|c|c|c|}
\hline \multirow{3}{*}{ Variáveis } & \multicolumn{2}{|c|}{ Qualidade de sono } & \multirow{3}{*}{$\boldsymbol{P}$} \\
\hline & Ruim & Boa & \\
\hline & $n(\%)$ & $n(\%)$ & \\
\hline \multicolumn{4}{|l|}{ Idade } \\
\hline$\leq 25$ anos & $96(63,6)$ & $24(15,9)$ & \multirow{2}{*}{0,93} \\
\hline$>25$ anos & $25(16,6)$ & $6(4,0)$ & \\
\hline \multicolumn{4}{|l|}{ Sexo } \\
\hline Masculino & $31(20,5)$ & $13(8,6)$ & \multirow{2}{*}{0,06} \\
\hline Feminino & $90(54,6)$ & $17(11,3)$ & \\
\hline \multicolumn{4}{|l|}{ Frequênciade Atividade Física } \\
\hline Nenhuma vez por semana & $31(24,5)$ & $9(6,0)$ & \multirow{4}{*}{0,83} \\
\hline Menos de 1 vez por semana & $14(9,3)$ & $5(3,3)$ & \\
\hline 1 ou 2 vezes por semana & $27(17,9)$ & $7(4,6)$ & \\
\hline 3 ou mais vezes por semana & $43(28,5)$ & $9(6,0)$ & \\
\hline \multicolumn{4}{|l|}{ Ano do Curso } \\
\hline Primeiro & $53(35,1)$ & $13(8,6)$ & \multirow{4}{*}{0,66} \\
\hline Segundo & $24(15,9)$ & $5(3,3)$ & \\
\hline Terceiro & $14(9,3)$ & $6(4,0)$ & \\
\hline Quarto & $30(19,9)$ & $6(4,0)$ & \\
\hline
\end{tabular}

Fonte: Silvera ALOA, et al., 2021. 
Tabela 2 - Componentes apurados através do questionário PSQI.

\begin{tabular}{|c|c|}
\hline Componentes & $\mathrm{n}(\%)$ \\
\hline \multicolumn{2}{|l|}{ Qualidade subjetiva do sono } \\
\hline Muito boa & $9(6 \%)$ \\
\hline Boa & $74(49 \%)$ \\
\hline Ruim & $61(40,4 \%)$ \\
\hline Muito ruim & $7(4,6 \%)$ \\
\hline \multicolumn{2}{|l|}{ Latência do sono } \\
\hline$\leq 15 \min$ & $42(27,8 \%)$ \\
\hline 16 a $30 \mathrm{~min}$ & $44(29,1 \%)$ \\
\hline 31 a $60 \mathrm{~min}$ & $46(30,5 \%)$ \\
\hline$>60 \min$ & $19(12,6 \%)$ \\
\hline \multicolumn{2}{|l|}{ Duração do sono } \\
\hline$>7 \mathrm{~h}$ & $18(11,9 \%)$ \\
\hline 6 a $7 \mathrm{~h}$ & $86(57,0 \%)$ \\
\hline 5 a $6 \mathrm{~h}$ & $36(23,8 \%)$ \\
\hline$<5 \mathrm{~h}$ & $11(7,3 \%)$ \\
\hline \multicolumn{2}{|l|}{ Eficiência habitual do sono } \\
\hline$>85 \%$ & $109(72,2 \%)$ \\
\hline 75 a $84 \%$ & $25(16,6 \%)$ \\
\hline 65 a $74 \%$ & $9(5,9 \%)$ \\
\hline$<65 \%$ & $5(3,3 \%)$ \\
\hline \multicolumn{2}{|l|}{ Frequência de distúrbios do sono } \\
\hline $0^{*}$ & $5(3,3 \%)$ \\
\hline $1-9^{*}$ & $98(64,9 \%)$ \\
\hline $10-18^{*}$ & $41(27,2 \%)$ \\
\hline $19-27^{*}$ & $7(4,6 \%)$ \\
\hline \multicolumn{2}{|l|}{ Uso de medicação para dormir } \\
\hline Nenhuma vez por semana & $115(76,2 \%)$ \\
\hline Menos de uma vez por semana & $14(9,3 \%)$ \\
\hline Uma ou duas vezes por semana & $8(5,3 \%)$ \\
\hline Mais de três vezes na semana & $14(9,3 \%)$ \\
\hline \multicolumn{2}{|c|}{ Dificuldade de se manter acordado } \\
\hline Nenhuma vez por semana & $74(49 \%)$ \\
\hline Menos de uma vez por semana & $42(27,8 \%)$ \\
\hline Uma ou duas vezes por semana & $24(15,9 \%)$ \\
\hline Mais de três vezes na semana & $11(7,3 \%)$ \\
\hline \multicolumn{2}{|l|}{ Dificuldade de manter o ânimo } \\
\hline Nenhuma vez por semana & $14(9,3 \%)$ \\
\hline Menos de uma vez por semana & $54(35,8 \%)$ \\
\hline Uma ou duas vezes por semana & $51(33,1 \%)$ \\
\hline Mais de três vezes na semana & $32(21,2 \%)$ \\
\hline
\end{tabular}

Legenda: *Pontuação calculada pelo questionário.

Fonte: Silvera ALOA, et al., 2021.

\section{DISCUSSÃO}

De acordo com o PSQI, 79,48\% $(n=120)$ dos participantes em nosso estudo apresentavam qualidade ruim no padrão do sono. Usando o mesmo instrumento PSQI, muitos estudos anteriores e recentes em todo o mundo revelaram que uma alta porcentagem de estudantes de graduação em medicina sofre de sono insuficiente. Esse resultado, embora maior, vai de encontro com outros estudantes de medicina, que encontraram 64,5\%, e 76,3\% (SILVA RRP, et al., 2020; RIBEIRO CR, et al., 2014). Um estudo, também com estudantes de medicina de Botucatu, encontrou uma cifra ainda maior, representada por $87,1 \%$ (CORRÊA CC, et al., 2017).

O PSQI médio encontrado em nossa pesquisa foi de 8. Ferreira CMG et al. (2017) encontrou um PSQI médio de 7,2. Ambos são resultados bem acima da metanálise de Jahrami H, et al. (2020), cujo PSQI médio 
foi de 6,3. As altas taxas de sono ruim entre estudantes de medicina de diferentes locais são de grande preocupação, uma vez que podem ser trabalhadas através de medidas de alto custo-efetividade. Uma gama de repercussões fisiológicas e comportamentais podem ocorrer devido à baixa qualidade de sono, tais como redução na qualidade de vida e da eficiência acadêmica e profissional, culminando em um maior risco de acidentes profissionais e erro médico (MOREIRA et al., 2016).

Não foi encontrada associação significava entre sexo e qualidade de sono ( $p=0,06)$, tal como na pesquisa de Segundo LVG, et al. (2017) e Ferreira CMG, et al. (2017). Um estudo com estudantes de enfermagem encontrou pior qualidade do sono no sexo feminino. Apesar de não se saber por qual mecanismo isso ocorra, acredita-se que exista uma maior susceptibilidade ao estresse devido às variações hormonais e fisiológicas (SANTOS AF, et al., 2020). Do outro lado, nas pesquisas com resultado oposto, postula-se que a maior prevalência de vícios e abuso de drogas entre estudantes do sexo masculino seja uma justificativa para os resultados encontrados (GIRI PA, et al., 2013; CANDIDO FJ, et al., 2018). A definição de variáveis de risco para um sono de pior qualidade é fundamental para o estudo de estratégias direcionadas.

A prática de atividades físicas é associada a uma melhor saúde mental e melhor qualidade de sono, provavelmente devido à regulação do ritmo circadiano. Hoje, sabe-se que essas três variáveis estão interligadas, com alterações em cada uma repercutindo nas outras duas, como em um ciclo contínuo e vicioso (GHROUZ AK, et al., 2019).

Alguns modelos teóricos tentam explicar a relação benéfica de sono e exercício físico. O modelo termo regulatório teoriza que o aumento da temperatura corpórea resultante das atividades físicas favorece o gatilho inicial para o sono, além do aumento do sono de ondas lentas e do sono profundo, nos quais ocorre a recuperação física. O modelo da conservação de energia traz o sono como uma resposta para um balanço de energia gasto durante o exercício, preparando o corpo para as próximas horas acordado. A hipótese restaurativa afirma que o catabolismo resultante do exercício precisa ser contrabalanceado por um anabolismo, que ocorrerá durante as fases do sono (OLIVEIRA LMFT, et al., 2018).

A realização de atividades físicas não altera significativamente a quantidade diária de sono, mas possui efeito expressivo sobre a autopercepção dele, com melhora da qualidade de sono no PSQI. Sabe-se que a percepção ruim e a menor quantidade de horas de sono são fatores preditivos para desfechos adversos em saúde nos idosos. Isso sugere a necessidade de novos parâmetros para tratamento nos distúrbios relacionados ao sono (YOUNG PY, et al., 2012).

No presente estudo não houve relação estatística significativa entre realização de atividades físicas e qualidade de sono. Giri PA, et al. (2013) e Basu M, et al. (2019) encontraram relação entre hábito de atividades físicas e menor prevalência de distúrbios do sono. A diferença entre a avaliação de atividades físicas adotada neste estudo (avaliação quantitativa) e os apresentados (avaliação qualitativa) pode justificar a ausência da significância estatística

O consumo de drogas e substâncias psicoativas é um problema de saúde pública crescente dentro da população universitária, incluindo os estudantes de medicina. Numa metanálise, álcool e o tabaco foram as drogas mais consumidas pelos estudantes de medicina brasileiros. As drogas ilícitas mais usadas foram maconha, solventes, lança-perfume e os ansiolíticos. O sexo masculino apresentou maior proporção de consumo de drogas e foi encontrada maior prevalência de consumo ao longo do curso de medicina, o que pode ser resultado das crescentes exigências, fatores estressantes intrínsecos à graduação (ex. lidar com a morte e alterações do ciclo sono-vigília) e do declínio da saúde mental ao longo do curso (CANDIDO FJ, et al., 2018).

O uso de medicações hipnóticas, sobretudo de benzodiazepínicos, é preocupante devido à associação bem descrita entre efeitos colaterais perigosos, com sedação residual, prejuízos na memória, depressão do centro respiratório, quedas, acidentes de trânsito e de trabalho, insônia rebote, taquifilaxia e dependência (BACELAR A e JUNIOR LRP, 2019). No presente estudo, houve relação significativa entre uso de medicações e uma pior qualidade de sono $(p=0,006)$, sendo relatada por $23,9 \%$ dos participantes da pesquisa. Comparativamente, é uma porcentagem bem maior daquela observada em outros grupos de estudantes de medicina, como relatado por Ferreira CMG, et al. (2017): 16,8\%. 
A eficiência habitual do sono foi considerada adequada em $72,2 \%(n=109)$ dos participantes do presente estudo. Tal resultado é menor, porém semelhante ao encontrado em estudantes de medicina de Botucatu $(87,9 \%)$ e estudantes de enfermagem de São Paulo (85\%) (CORRÊA CC, et al., 2017; SALVI CCP, et al., 2020). Quanto à qualidade subjetiva do sono, $45 \%$ da amostra classificou-o como ruim ou muito ruim. Isso é particularmente interessante, uma vez que o PSQI aponta uma qualidade de sono ruim de quase $80 \%$, refletindo uma provável negligência dos estudantes acerca de seu próprio padrão de sono. Isso foi observado por outro estudo (SILVA RRP, et al., 2020).

Curiosamente, estudantes de medicina apresentam melhores práticas de higiene do sono do que estudantes de outros cursos. Isso se deve provavelmente a um maior conhecimento sobre importância do sono e exaustiva carga horária da graduação em Medicina (AL-KANDARI S, et al., 2017). Estariam os estudantes iludindo-se acerca da própria qualidade do sono apenas por adotarem mais práticas de higiene de sono em relação aos outros cursos?

Não houve relação entre $o$ ano da graduação e a qualidade do sono ( $p>0,05)$. Nas outras pesquisas analisadas, a graduação geralmente foi dividida em períodos de ciclo básico, ciclo clínico e internato. Santos $A F$, et al. (2020), em sua pesquisa com estudantes de enfermagem, encontrou pior qualidade de sono em semestres mais avançados, descartando a importância dos rodízios de turnos (inclusive noturnos) e maior exposição a fatores estressores da prática clínica, como comunicação multiprofissional, aperfeiçoamento de habilidades e os sofrimentos que surgem junto do cuidado.

Entre estudantes de medicina, os dados apontam para pior qualidade de sono entre aqueles que estão iniciando a faculdade, ou seja, estão no ciclo básico. Isso talvez possa ser explicado pelas menores capacidades de enfrentamento, ausência de independência ou autonomia em relação a estudantes de fases mais avançadas, distanciamento de família e amigos, necessidade de realizar tarefas e funções independentemente, bem como maior carga de estudos em relação ao ensino médio e ansiedade ao lidar com sua primeira profissão. (GHROUZ AK, et al., 2019; BASU M, et al., 2019). Do outro lado, Giri PA, et al. (2013) encontrou mais distúrbios do sono nos pós-graduados, provavelmente devido à necessidade de lidar com responsabilidades do cuidado ao paciente.

A duração média do sono dentre os estudantes de medicina é variável. Na Arábia Saudita, estudantes do $4^{\circ}$ ao 6ํㅜㄹ ano dormiam em média 5,8 horas (ALSAGGAF MA, et al., 2016). Numa metanálise incluindo 43 estudos com estudantes de medicina, a média de sono diário ficou em 6,3 horas, com uma menor prevalência de sono ruim dentre os estudantes brasileiros, contudo, estes apresentaram maior cansaço e sonolência diurnos excessivos (JAHRAMI H, et al., 2020).

Na presente pesquisa, a duração média do sono girou em torno de 6,7horas, valor pouco abaixo do recomendado pelos consensos de insônia, que recomendam de 7-9 horas de sono para adultos jovens (WATSON NF, et al., 2015). Chama atenção a alta prevalência de sono ruim apesar deste achado, sinalizando a necessidade pesquisas mais aprofundadas acerca dos distúrbios específicos que estão afetando o sono dos estudantes.

O estudo em questão apresenta algumas limitações. Uma delas é ser um estudo transversal. Um estudo longitudinal seria mais fidedigno, uma vez que a qualidade de sono pode variar conforme período de provas. Outra limitação refere-se ao contexto de pandemia, a qual mudou drasticamente os hábitos de sono e de vida dos estudantes. Para contornar isso, pediu-se aos mesmos que respondessem no questionário de acordo os seus padrões de sono 1 mês antes do início da quarentena. O PSQI é validado para a qualidade de sono do mês anterior e não períodos mais distantes de tempo, uma vez que pode ser mais difícil do estudante se lembrar de seus hábitos com o passar do tempo (viés de memória).

Além disso, a amostragem reduzida da presente pesquisa limitou a significância estatística de muitas das variáveis estudadas. Os resultados de um único centro universitário não podem ser generalizados para a população geral. Estudos futuros longitudinais, com amostragens maiores e diagnóstico preciso dos distúrbios do sono responsáveis são necessários. Por último, estudos baseados em avaliação subjetiva estão mais predispostos às informações incorretas fornecidas pelos próprios objetos do estudo, além da possibilidade de interpretações erradas acerca do questionário. 


\section{CONCLUSÃO}

Com base nos achados encontrados no presente estudo, pode-se evidenciar que a autoavaliação do sono entre os estudantes de Medicina é precária, com quase $80 \%$ da amostra apresentando uma qualidade de sono ruim. Das variáveis estudadas, apenas o uso de medicação apresentou significância estatística com sono ruim, não sendo possível inferir relação de causalidade por se tratar de um estudo transversal. Percebese que, apesar da extensa literatura abrangendo a qualidade de sono nessa população, pouco se faz na prática para combater esse sério problema. A má qualidade de sono continua sendo negligenciada principalmente pelos próprios estudantes, que não se reconhecem como população de risco.

\section{REFERÊNCIAS}

1. AL-KANDARI S, et al. Association between sleep hygiene awareness and practice with sleep quality among Kuwait University students. Sleep health, 2017; 3(5): 342-347.

2. ALSAGGAF MA, et al. Sleep quantity, quality, and insomnia symptoms of medical students during clinical years. Relationship with stress and academic performance.Saudi Med J, 2016; 37(2): 173-182.

3. BACELAR A, JUNIOR LRP. Insônia: do diagnóstico ao tratamento. São Caetano do Sul: Difusão Editora, $2019 ; 173 p$.

4. BASU M, et al. A Study on Sleeping Pattern among Undergraduate Medical Students of a Tertiary Care Teaching Hospital of Kolkata. International Journal of Medicine and Public Health, 2019; 9(4): 118-124.

5. BUYSSE DJ, et al. The Pittsburgh Sleep Quality Index: A New Instrument for Psychiatric Practice and Research Psychiatry Research, 1989; 28: 193-213.

6. CANDIDO FJ, et al. The use of drugs and medical students: a literature review. Rev. Assoc. Med. Bras, 2018; 64(5): 462-468.

7. CHAZAN ACS, et al. Qualidade de vida de estudantes de medicina da UERJ por meio do Whoqol-bref: uma abordagem multivariada. Ciência \& Saúde Coletiva, 2015; 20: 547-556

8. CORRÊA CC, et al. Qualidade de sono em estudantes de medicina: comparação das diferentes fases do curso. J Bras. Pneumol, 2017; 43: 285-289.

9. D'AUREA C, et al. Objective short sleep duration is associated with the activity of the hypothalamic-pituitary-adrenal axis in insomnia. Arquivos de neuro-psiquiatria, 2015; 73(6): 516-519.

10. FERREIRA CMG, et al. Qualidade do sono em estudantes de medicina de uma universidade do Sul do Brasil. Conexão Ciência, 2017; $12(1)$ : 78-85.

11. GHROUZ AK, et al. Physical activity and sleep quality in relation to mental health among college students. Sleep and Breathing, 2019; 23(2): 627-634.

12. GIRI PA, et al. Study of Sleep Habits and Sleep Problems Among Medical Students of Pravara Institute of Medical Sciences Loni, Western Maharashtra, India. Ann Med Health Sci, 2013; 3: 51-54.

13. HIROTSU C, et al. Sleep complaints in the Brazilian population: impact of socioeconomic factors. Sleep Sci, 2014; 7 : 135-142.

14. JAHRAMI H, et al. Prevalence of sleep problems among medical students: a systematic review and meta-analysis. J Public Health (Berl), 2020; 28: 605-622.

15. MOREIRA APF, et al. Avaliação da qualidade de vida, sono e Síndrome de Burnout dos residents de um programa de residência multiprofissional em saúde. Revista Medicina Ribeirão Preto, 2016; 49(5): 393-402.

16. OKANO K, et al. Sleep quality, duration, and consistency are associated with better academic performance in college students. NPJ science of learning, 2019; 4(1): 1-5.

17. OLIVEIRA LMFT, et al. Exercise or Physical Activity: Which is more strongly associated with the perception of sleep quality by adolescentes?. Rev. paul. pediatria, 2018; 36(3): 322-328.

18. SALVI CPP, et al. Profile of nursing students: quality of life, sleep and eating habits. Revista Brasileira de Enfermagem, $2020 ; 73$.

19. SANTOS AFD, et al. Qualidade do sono e fatores associados em universitários de enfermagem. Acta paul. enferm. 2020; 33: 1-8.

20. SEGUNDO LVG, et al. Aspectos relacionados à qualidade do sono em estudantes de medicina / features related to quality of sleep in medical students. Revista Brasileira de Neurologia e Psiquiatria, 2017; 21: 213-223.

21. SILVA RRP, et al. Qualidade do sono e sonolência excessiva entre estudantes de medicina. Revista de Medicina, 2020; 99(4): 350-356.

22. RIBEIRO CR, et al. The impact of sleep quality in medical. Rev Soc Bras Clin Med, 2014: 12(1): 8-14.

23. WATSON NF, et al. Recommended Amount of Sleep for a Healthy Adult: A Joint Consensus Statement of the American Academy of Sleep Medicine and Sleep Research Society. Journal of Clinical Sleep Medicine, 2015; 11(6): 591-592.

24. YANG PY, et al. Exercise training improves sleep quality in middle-aged and older adults with sleep problems: a systematic review. J Physiother, 2012: 58(3):157-63. 